\title{
OPTIMAL PRESSURE BOUNDARY CONTROL OF STEADY MULTISCALE FLUID-STRUCTURE INTERACTION SHELL MODEL DERIVED FROM KOITER EQUATIONS
}

\section{ANDREA CHIERICI ${ }^{1}$, LEONARDO CHIRCO ${ }^{1}$, VALENTINA GIOVACCHINI ${ }^{1}$, SANDRO MANSERVISI ${ }^{1}$ AND EMANUELA MARZI ${ }^{1}$}

\author{
${ }^{1}$ University of Bologna - DIN \\ Via dei Colli 16, 40136 Bologna (BO), Italy \\ e-mail: andrea.chierici4@unibo.it
}

Key words: Optimal Boundary Control, Adjoint Variables, Multiscale FSI

\begin{abstract}
The fluid-structure interaction (FSI) problem has been extensively studied, and many papers and books are available in the literature on the subject. In this work, we consider some optimal FSI pressure boundary control applications by using a membrane model derived from the Koiter shell equations where the thickness of the solid wall can be neglected and the computational cost of the numerical problem reduced. We study the inverse problem with the aim of achieving a certain objective by changing some design parameters (e.g. forces, boundary conditions or geometrical domain shapes) by using an optimal control approach based on Lagrange multipliers and adjoint variables. In particular, a pressure boundary optimal control is presented in this work. The optimality system is derived from the first-order optimality condition by taking the Fréchet derivatives of the Lagrangian with respect to all the variables involved. This system is solved by using a finite element code with mesh-moving capabilities. In order to support the proposed approach, we perform numerical tests where the pressure on a fluid domain boundary controls the displacement that occurs in a well-defined region of the solid domain.
\end{abstract}

\section{Introduction}

Recently, the numerical simulations of fluid-structure interaction (FSI) problems have become more and more popular, and many papers and books have been published on this topic (see $[1,2,3,4,5])$. The applications of the numerical modeling of FSI systems are various, raging from wind turbines and aircraft to hemodynamics. In FSI problems the fluid flow changes the tensional state of a solid structure that is left free to move and the solid deformation has an important effect on the fluid flow.

Several techniques have been developed to reduce the computational cost of FSI problems. In this respect, this work is based on the reduction of the dimensionality of the solid, through a model built on the Koiter shell equations [6]. In order to couple the fluid and the structure domains, the Koiter shell equations are embedded into the fluid equations as a Robin boundary condition [7]. The coupling fluid-structure conditions are automatically treated implicitly, so the stability of this numerical scheme is preserved. This model has many applications in cases where a fluid interacts with a thin membrane that deforms mainly in the normal direction. 
In the last years optimization problems have gained popularity among the research community by using gradient-based adjoint methods, see [8,9]. For adjoint FSI optimization the interested reader can see [10, $11,12,13]$ and the references therein. In this paper, we solve a stationary displacement matching problem where the control variable is the fluid boundary pressure using the Lagrangian multipliers method to obtain the optimality system. The rest of this paper is organized as follows. In Section 2 we introduce the mathematical model describing our multi-scale FSI problem and in Section 3 we derive the optimality system arising from the minimization of the augmented Lagrangian is presented. In Section 4 some simple two-dimensional numerical results are then reported.

\section{Physical model}

In this section we introduce the mathematical model for the FSI problem. We consider the classic NavierStokes equations to model the fluid motion, and a shell model to describe the solid behavior. In particular, the structural model is based on the Koiter shell approach that considers the model of an elastic thin membrane. We introduce now some functional spaces defined on the domain $\Omega$ used in the rest of the paper: we denote with $L^{2}(\Omega)$ the space of square integrable functions, and with $H^{s}(\Omega)$ the standard Sobolev space with norm $\|\cdot\|_{s}$. Moreover, we denote with $H_{0}^{s}(\Omega)$ the space of all functions in $H^{s}(\Omega)$ that vanish on the boundary of $\Omega$.

The Koiter shell approach relies on the assumptions that the structure displacements are small and normal

to the shell surface. The domain of the structure is denoted by $\Gamma_{s}$, the displacement and the external

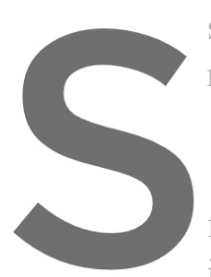
surface force vectors b

results

for appropriate test functions $\psi$ belonging to a fu imposed boundary conditions.
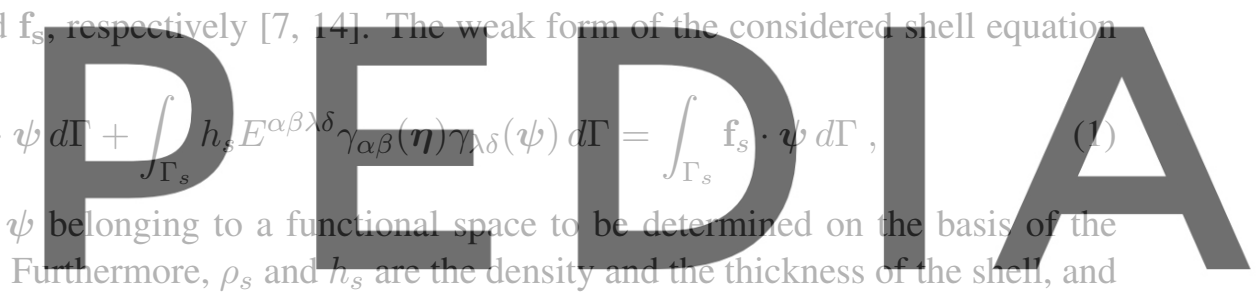

$E^{\alpha \beta \lambda \delta}$ and $\gamma_{\alpha \beta}$ are the elasticity and the change of metric, respectively.

Register for free at https/(www.scipedia.com to download the version without the watermark

isotropic material are considered [6]. Under these hypotheses the structure model (1) reduces to a simple

scalar equation. The dimension of the structure is then reduced by one. So the following simplified model is obtained

$$
\begin{aligned}
& \rho_{s} h_{s} \frac{\partial^{2} \boldsymbol{\eta}_{3}}{\partial t^{2}}+\beta \boldsymbol{\eta}_{3}=f_{s} \quad \text { on } \quad \Gamma_{s} \text {, } \\
& \left.\boldsymbol{\eta}_{3}\right|_{t=0}=\boldsymbol{\eta}_{0},\left.\quad \frac{\partial \boldsymbol{\eta}_{3}}{\partial t}\right|_{t=0}=\boldsymbol{\eta}_{v} \quad \text { on } \quad \Gamma_{s},
\end{aligned}
$$

where $\rho_{f}$ and $\mathbf{u}$ are the density and the velocity vector of the fluid, respectively, and $\boldsymbol{\eta}_{3}$ represents the displacement normal to the reference solid surface. In particular, when one considers cylindrical geometries of radius $R$, it can be demonstrated that [7]

$$
\beta=\frac{h_{s} E}{1-\nu^{2}} \frac{1}{R^{2}},
$$


The fluid is modeled as Newtonian, homogeneous and incompressible, described in ALE form as $[2,15]$

$$
\left.\rho_{f} \frac{\partial \mathbf{u}}{\partial t}\right|_{\mathcal{A}}+\rho_{f}[(\mathbf{u}-\mathbf{w}) \cdot \nabla] \mathbf{u}-\nabla \cdot \boldsymbol{\sigma}^{f}=\mathbf{0} \quad \text { on } \quad \Omega_{f},
$$

where $\rho_{f}$ and $\mathbf{u}$ are the density and the velocity vector of the fluid. Moreover, $\boldsymbol{\sigma}^{f}$ is the Cauchy stress tensor of the fluid written as $\boldsymbol{\sigma}^{f}=-p \mathbf{I}+\mu\left(\nabla \mathbf{u}+\nabla \mathbf{u}^{T}\right), \mu$ and $p$ are the dynamic viscosity and the pressure of the fluid, respectively. The system of equations (4) is completed with appropriate boundary conditions. The fluid domain is $\Omega_{f}$, and $\mathbf{w}$ is the ALE velocity that determines step by step the position of the nodes of the fluid domain as $\mathbf{x}_{f}(t)=\mathbf{x}_{0}+\int_{0}^{t} \mathbf{w} d \tau$.

This shell model allows us to reduce by one the dimension of the solid, so the structural equations can be reduced to a boundary condition on $\Gamma_{s}$ for the fluid problem. The two sub-systems (4) and (2) can be coupled by imposing $\boldsymbol{\sigma}^{f} \cdot \mathbf{n}-f_{s}=0$ on $\Gamma_{s}$. We define now the functional space $V^{0}=\left\{\phi \in H^{1}\left(\Omega_{f}\right)\right.$ : $\left.\left.\boldsymbol{\phi}\right|_{\Gamma_{D, f}}=\mathbf{0}\right\}$, where $\Gamma_{D, f}$ are the boundaries of $\Omega_{f}$ where a Dirichlet condition is imposed. In order to satisfy the continuity of the test functions $\phi \cdot \mathrm{n}=\psi$ over the interface surface $\Gamma_{s}$ in the coupled system, we introduce the following functional space

$$
W^{0}=\left\{(\phi, \psi) \in V^{0} \times H^{1}\left(\Gamma_{s}\right): \phi \cdot \mathbf{n}=\psi \text { over } \Gamma_{s}\right\}
$$

Now we can derive the weak form of the coupled final system
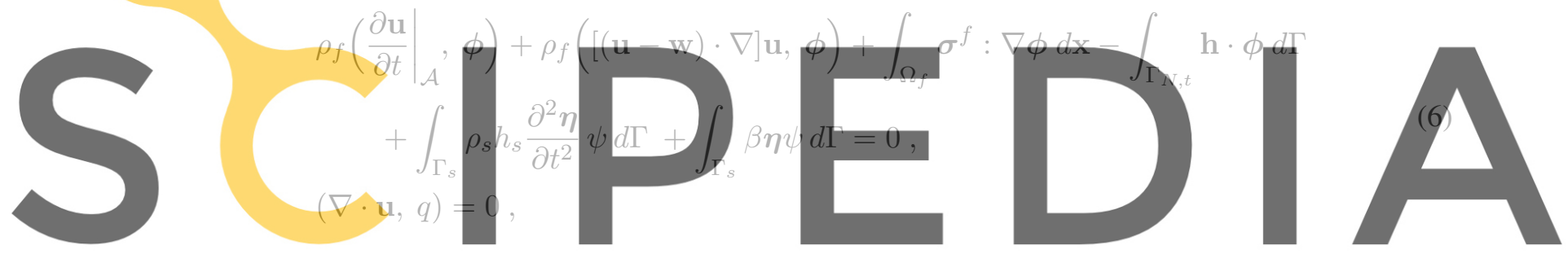

for all $(\phi, \psi) \in W^{0}, q \in L^{2}\left(\Omega_{f}\right)$. A finite element technique is used to obtain the discrete weak

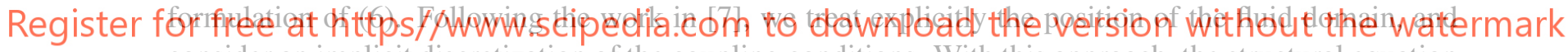
consider an implicit discretization of the coupling conditions. With this approach, the structural equation can be incorporated into the fluid equations as a boundary condition (Robin scheme).

However, this work is based on the stationary solution of the presented system. Under this hypothesis, the system (6) becomes

$$
\begin{aligned}
& \rho_{f}((\mathbf{u} \cdot \nabla) \mathbf{u}, \boldsymbol{\phi})+\int_{\Omega_{f}} \boldsymbol{\sigma}^{f}: \nabla \boldsymbol{\phi} d \mathbf{x}-\int_{\Gamma_{N, t}} \mathbf{h} \cdot \boldsymbol{\phi} d \Gamma+\int_{\Gamma_{s}} \beta \boldsymbol{\eta} \psi d \Gamma=0, \\
& (\nabla \cdot \mathbf{u}, q)=0 .
\end{aligned}
$$

\section{Optimality system}

In this work, we are interested in solving a given shell deformation by controlling the fluid pressure over a boundary. For this purpose, we start introducing the following objective functional

$$
\mathcal{J}(\boldsymbol{\eta}, p)=\frac{1}{2} \int_{\Gamma_{d}}\left\|\boldsymbol{\eta}-\boldsymbol{\eta}_{d}\right\|^{2} d \Gamma+\frac{\lambda}{2} \int_{\Gamma_{c}} p^{2} d \Gamma
$$


where the first term is the distance in norm between the actual displacement and the desired value over the controlled boundary $\Gamma_{d}$, and the second term is a standard Tychonov regularization term that limits the $L^{2}$-norm of the fluid boundary pressure $p_{c}$, i.e. the control variable. The regularization parameter $\lambda$ weights the importance of the two terms over the cost functional. In general, too much regularization leads to smoother but less effective controls, while a lack of regularization may cause numerical issues since usually the optimal solution lies in distributional spaces.

We now introduce the following augmented Lagrangian functional $\mathcal{L}$, that is obtained by adding to the objective functional $\mathcal{J}$ the FSI state equations (7) multiplied by a set of Lagrange multipliers. In the following, we refer to the Lagrange multipliers as adjoint variables.

$$
\begin{aligned}
& \mathcal{L}\left(\boldsymbol{\eta}, \mathbf{u}, \mathbf{u}_{a}, p, p_{a}, \Gamma\right)=\mathcal{J}(\boldsymbol{\eta}, p)-\left(\rho^{f}(\mathbf{u} \cdot \nabla) \mathbf{u}, \mathbf{u}_{a}\right)+\left(p, \nabla \cdot \mathbf{u}_{a}\right)-\left(p_{a}, \nabla \cdot \mathbf{u}\right) \\
& \quad-\mu\left(\nabla \mathbf{u}, \nabla \mathbf{u}_{a}\right)+\int_{\Gamma} \mu(\nabla \mathbf{u} \cdot \mathbf{n}) \cdot \mathbf{u}_{a} d \Gamma-\int_{\Gamma}(p \mathbf{n}) \cdot \mathbf{u}_{a} d \Gamma-\int_{\Gamma_{t}} \mathbf{u}_{a} \cdot\left(\beta \boldsymbol{\eta}-\mathbf{f}_{s}-\boldsymbol{\tau}_{n}\right) d \Gamma,
\end{aligned}
$$

where we integrate by parts the contributions of the fluid stress tensor $\sigma^{f}$. The surface integrals can be rewritten by substituting the definition of $\tau_{n}$. The stationary points of the Lagrangian functional can be found by setting to zero the Fréchet derivatives taken for all the problem variables. When the derivatives are taken with respect to the adjoint variables the weak form of the state system (7) is recovered as well as the boundary conditions. By taking the derivatives in the direction $\delta p$ we get

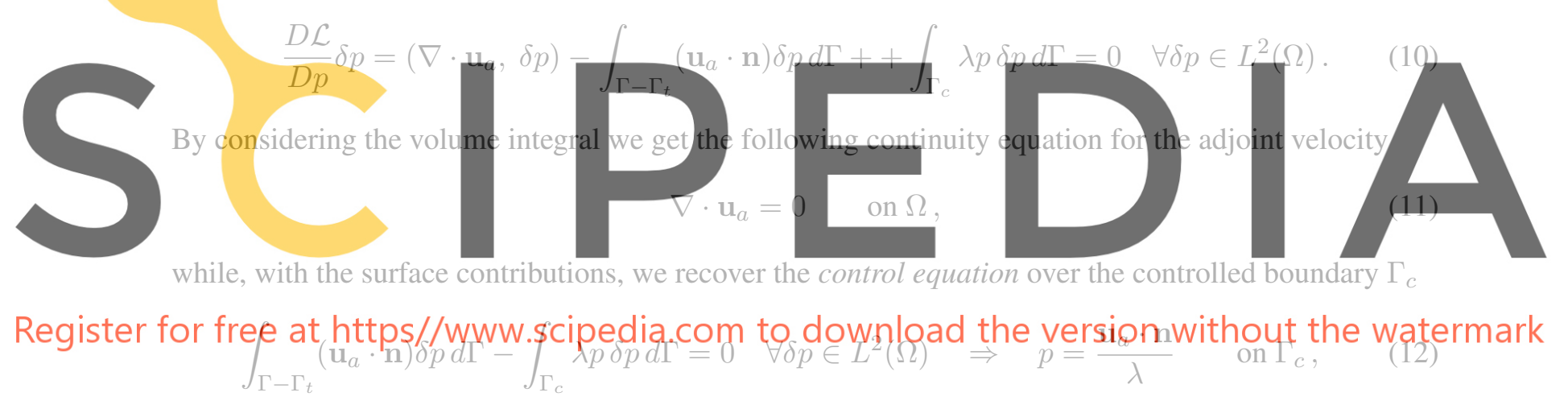

and the boundary conditions on $\Gamma_{D}$

$$
\int_{\Gamma-\Gamma_{t}-\Gamma_{c}}\left(\mathbf{u}_{a} \cdot \mathbf{n}\right) \delta p d \Gamma=0 \quad \forall \delta p \in L^{2}(\Omega) \quad \Rightarrow \quad \mathbf{u}_{a} \cdot \mathbf{n}=0 \quad \text { on } \Gamma_{D},
$$

On $\Gamma_{N}$ we have $\delta p=0$ since we prescribe Neumann boundary conditions with fixed pressure.

For $\delta \boldsymbol{\eta}$ we have

$$
\frac{D \mathcal{L}}{D \boldsymbol{\eta}} \delta \boldsymbol{\eta}=-\int_{\Gamma_{t}} \mathbf{u}_{a} \cdot \beta \delta \boldsymbol{\eta} d \Gamma+\int_{\Gamma_{d}}\left(\boldsymbol{\eta}-\boldsymbol{\eta}_{d}\right) \cdot \delta \boldsymbol{\eta} d \Gamma=0 \quad \forall \delta \boldsymbol{\eta} \in W^{0}
$$

Recalling that $\Gamma_{d} \subset \Gamma_{t}$ we obtain the following boundary conditions for the adjoint system

$$
\begin{array}{rlrl}
\mathbf{u}_{a} \beta-\left(\boldsymbol{\eta}-\boldsymbol{\eta}_{d}\right)=0 & & \text { on } \Gamma_{d}, \\
\mathbf{u}_{a} \beta & =0 & & \text { on } \Gamma_{t} .
\end{array}
$$


We collect $\delta \mathbf{u}$ terms and integrate by parts to obtain

$$
\begin{aligned}
& \rho^{f}\left([(\delta \mathbf{u} \cdot \nabla) \mathbf{u}+(\mathbf{u} \cdot \nabla) \delta \mathbf{u}], \mathbf{u}_{a}\right)-\left(\nabla p_{a}, \delta \mathbf{u}\right)-\mu\left(\nabla^{2} \mathbf{u}_{a}, \delta \mathbf{u}\right)+\int_{\Gamma}\left(p_{a} \mathbf{n}\right) \cdot \delta \mathbf{u} d \Gamma \\
& \quad-\int_{\Gamma-\Gamma_{t}} \mu(\nabla \delta \mathbf{u} \cdot \mathbf{n}) \cdot \mathbf{u}_{a} d \Gamma+\int_{\Gamma} \mu\left(\nabla \mathbf{u}_{a} \cdot \mathbf{n}\right) \cdot \delta \mathbf{u} d \Gamma=0 \quad \forall \delta \mathbf{u} \in W^{0} .
\end{aligned}
$$

The strong form of the adjoint velocity reads

$$
\rho^{f}(\nabla \mathbf{u})^{T} \cdot \mathbf{u}_{a}+\rho^{f}(\mathbf{u} \cdot \nabla) \mathbf{u}_{a}-\nabla p_{a}-\mu \nabla^{2} \mathbf{u}_{a}=0 \quad \text { on } \Omega,
$$

with boundary conditions

$$
\mathbf{u}_{a}=0 \quad \text { on } \Gamma_{D}, \quad \boldsymbol{\tau}_{n a}=0 \quad \text { on } \Gamma_{N} \cup \Gamma_{t} .
$$

Moreover we have to consider the contribution on $\mathcal{L}$ given by the motion of the boundary $\Gamma_{t}$ along the direction $\delta \eta$

$$
\frac{D \mathcal{L}}{D \Gamma} \delta \boldsymbol{\eta}=\int_{\Gamma_{t}} \beta\left(\nabla \mathbf{u}_{a} \cdot \mathbf{n}+\chi \mathbf{u}_{a}\right) \cdot \delta \boldsymbol{\eta} d \Gamma=0 \quad \forall \delta \boldsymbol{\eta} \in W^{0},
$$

where $\chi$ represents the shell curvature. Under the hypothesis of small deformation we can safely neglect the terms where $\chi$ appears. We also have that $\frac{D \mathcal{L}}{D \Gamma} \delta \boldsymbol{\eta}=0$ since the term with $\mathbf{u}_{a}$ is defined on the surface $\Gamma_{t}$, and a constant extension of it towards the normal direction to the surface leads to a null normal

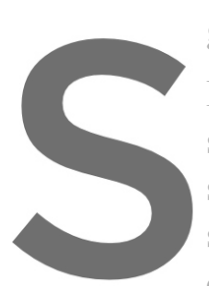
gradient of this term.

In short the optimality

system (11)-(16) and the

state variables the use of

solution of the state, adjoin
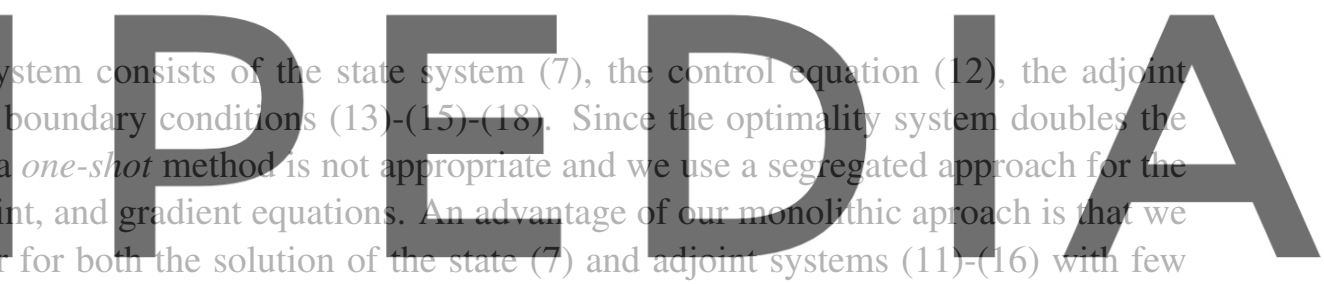

modifications

Register for free at https//www.scipedia.com to download the version without the watermark

\section{Numerical results}

In this section, we report some numerical results obtained by using the mathematical model shown in the previous sections. We consider a rectangular domain $\Omega=\{(x, y): x \in[0,0.1], y \in[0,0.3]\}$ as shown in Figure 1 on the left. The fluid has density $\rho^{f}=1000 \mathrm{~kg} / \mathrm{m}^{3}$ and dynamic viscosity $\mu=100 \mathrm{~Pa} \cdot \mathrm{s}$. For the solid, we consider $\beta=60 \mathrm{kPa} / \mathrm{m}$ and thickness $h_{s}=0.0075 \mathrm{~m}$. For all the presented simulations, the domain was uniformly divided with a regular rectangular mesh.

We implement a standard steepest descent algorithm in the multigrid finite element code FEMuS, that relies on PETSc libraries for the solution of the multigrid discretized linear solver with MPI paralellization.

\subsection{Plane channel test}

In this first test, the fluid flows vertically from the bottom to the top. The region of the boundary $\Gamma_{2}$ represents a solid wall with no-slip boundary condition $(\mathbf{u}=\mathbf{0})$ and $\Gamma_{3}$ is the membrane where we 
impose the generalized Robin boundary condition (2). In Figure 1, we also report the results obtained simulating the system without control and in steady-state. The displacement of the points in the domain (center) and the pressure (right) are shown. The pressure presents a linearly decreasing trend from the bottom, where $p=6000 P a$ is imposed, to the top, where we fixed $p=0 P a$, and it is shown with lines of iso-magnitude.
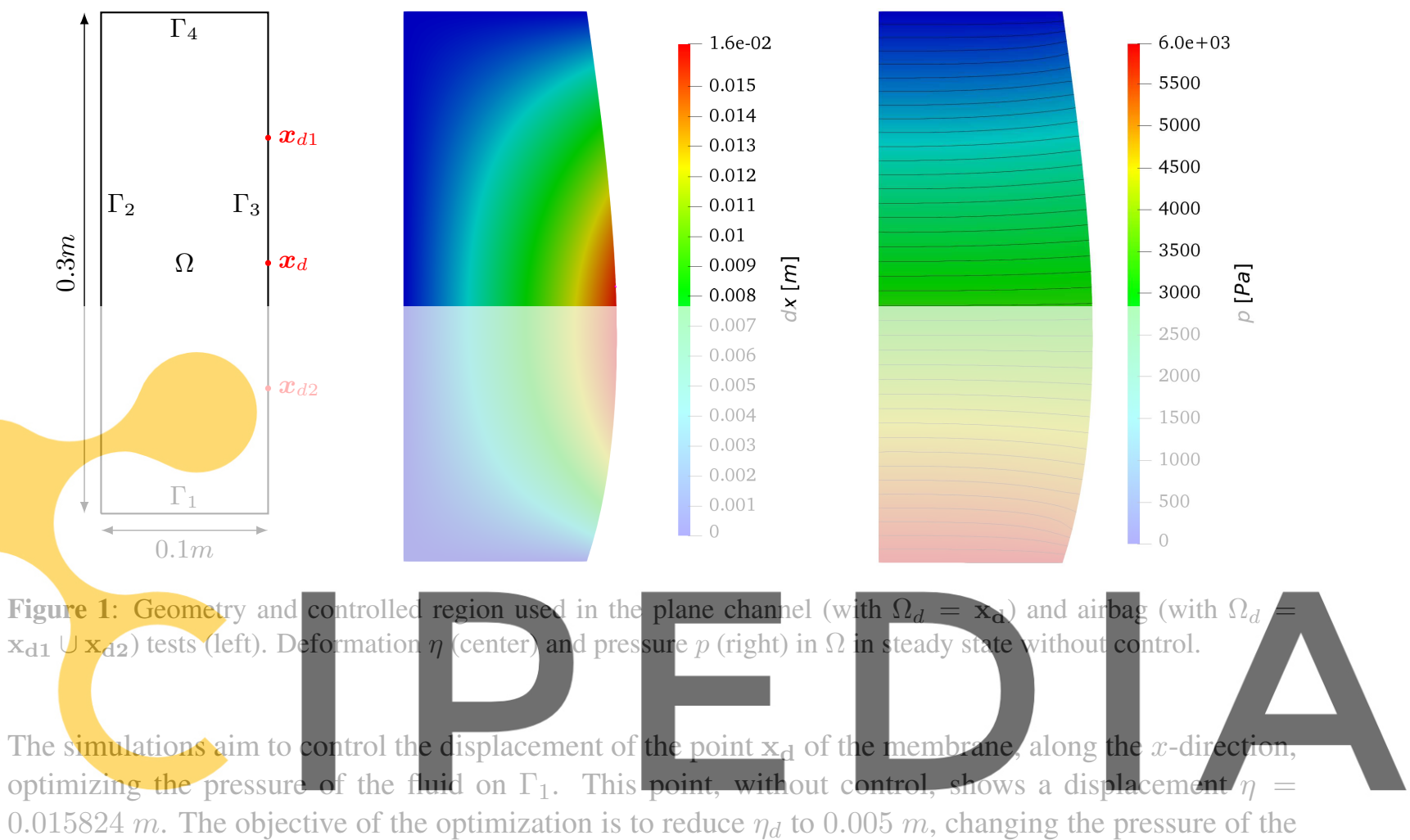

$0.015824 \mathrm{~m}$. The objective of the optimization is to reduce $\eta_{d}$ to $0.005 \mathrm{~m}$, changing the pressure of the

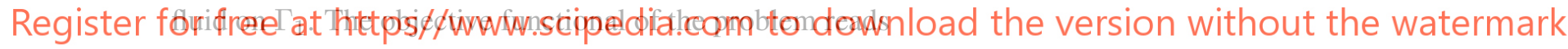

$$
\mathcal{J}(\eta, p)=\frac{1}{2}\left(\left.\eta\right|_{\mathbf{d}}-\eta_{d}\right)^{2}+\frac{\lambda}{2} \int_{\Gamma_{1}} p^{2} d \Gamma
$$

We solve multiple simulations with different regularization parameter $\lambda$. The results are presented in Table 1. Note that the smaller is $\lambda$, the closer the displacement of the controlled point $\mathbf{x}_{\mathbf{d}}$ is to the desired one. This result is expected, since with larger $\lambda$ the contribution of the regularization term in the minimization of the functional is more relevant. This is introduced in the functional in order to limit the control parameter $p$ to the space of square integrable functions. Therefore, with larger $\lambda$ we find more regular optimization parameter $p$, but less precise displacement field $\eta$. In Table, we also report the number of iterations needed for the implemented algorithm to find the optimal solution.

We focus now on the controlled inlet pressure field updated through the formula

$$
p_{c}^{i}=p_{c}^{i-1}-r^{i, j}\left(p_{c}^{i-1}-\frac{\mathbf{u}_{a}^{i} \cdot \mathbf{n}}{\lambda}\right) .
$$


Table 1: Objective functional $\mathcal{J}$, displacement $\eta$ and number of iteration obtained with different $\lambda$ values.

\begin{tabular}{cccc}
\hline$\lambda$ & $\mathcal{J}\left(\eta, p_{c}\right)$ & $\eta_{\text {opt }}[m]$ & Iterations \\
\hline$\infty$ & $5.85839 \cdot 10^{-05}$ & 0.015824 & - \\
$10^{-08}$ & $2.19246 \cdot 10^{-06}$ & 0.002906 & 4 \\
$10^{-09}$ & $5.54438 \cdot 10^{-09}$ & 0.004895 & 8 \\
$10^{-10}$ & $2.18941 \cdot 10^{-10}$ & 0.004979 & 10 \\
$10^{-11}$ & $6.10506 \cdot 10^{-12}$ & 0.004997 & 12 \\
$10^{-12}$ & $3.86734 \cdot 10^{-15}$ & 0.005000 & 26 \\
\hline
\end{tabular}

In fact, depending on the regularization parameter, different inlet pressure fields can be obtained. In Figure 2, the controlled pressure field along the boundary $\Gamma_{1}$ is reported for various values of $\lambda$. Note that the choice of the regularization parameter strongly affects the controlled pressure field. With weak regularization, the objective term dominates in the functional and the pressure attains large values, thus effectively controlling the membrane displacement. In Figure 2 it is also reported the reference starting pressure. The comparison between the uncontrolled field and all the controlled pressure fields show that the control strongly affects the solution on $\Gamma_{c}=\Gamma_{1}$ in order to obtain the desired displacement $\eta_{d}$. Moreover, in Figure 2 on the right the velocity field for $\lambda=10^{-15}$ is reported. Note that an inversion of the fluid flow in the inlet occurs, needed for reducing the displacement of the point $\mathrm{x}_{\mathrm{d}}$.
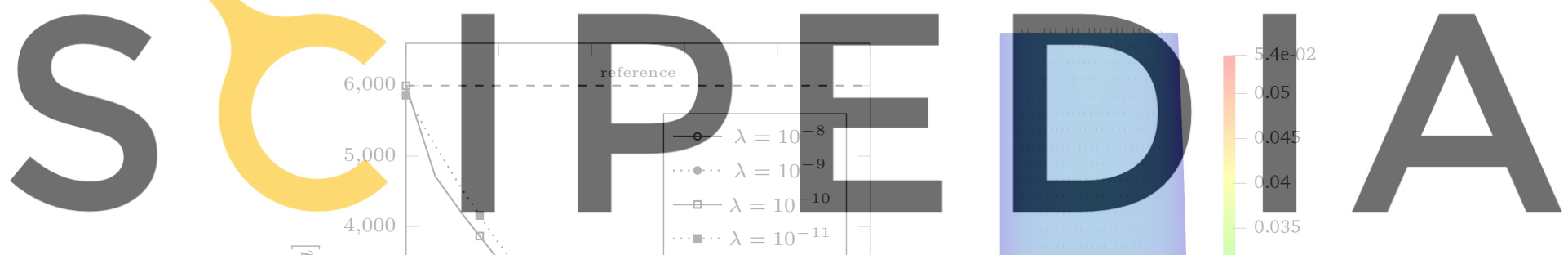

Register for free at htthps//www:scipedia.com to download the version without the watermark
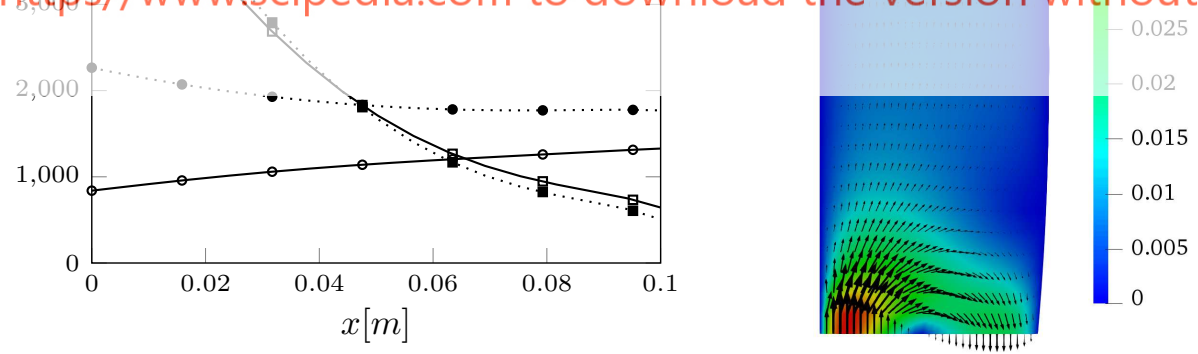

Figure 2: Plane channel test: control pressure $p$ on $\Gamma_{1}$ with different regularization parameters (left). The dotted line represents the pressure in the reference case with no control (i.e. $\lambda=\infty$ ). On the right: velocity $\mathbf{u}$ in $\Omega$ for $\lambda=10^{-15}$. 


\subsection{Airbag test}

We consider the same geometry of the previous test, togheter with airbag-like boundary conditions: we impose a no-slip condition on $\Gamma_{1} \cup \Gamma_{4}$ and the Koiter boundary condition on $\Gamma_{3}$. The controlled pressure is imposed on $\Gamma_{c}=\Gamma_{2}$, where we initially impose $p=300 P a$. We also consider all the physical properties introduced above. In this framework we want to control the displacement field on the two different points, $\boldsymbol{x}_{d 1}$ and $\boldsymbol{x}_{d 2}$ (see Figure 1, left).

Observing the results obtained in a steady state without control, shown in Figure 3, one can see that the problem is symmetric since we are neglecting the buoyancy forces. The two points $\mathbf{x}_{\mathbf{d}_{1}}$ and $\mathbf{x}_{\mathbf{d}_{2}}$ present a displacement $\eta=0.001152 \mathrm{~m}$ and the pressure in the channel is almost constant everywhere.
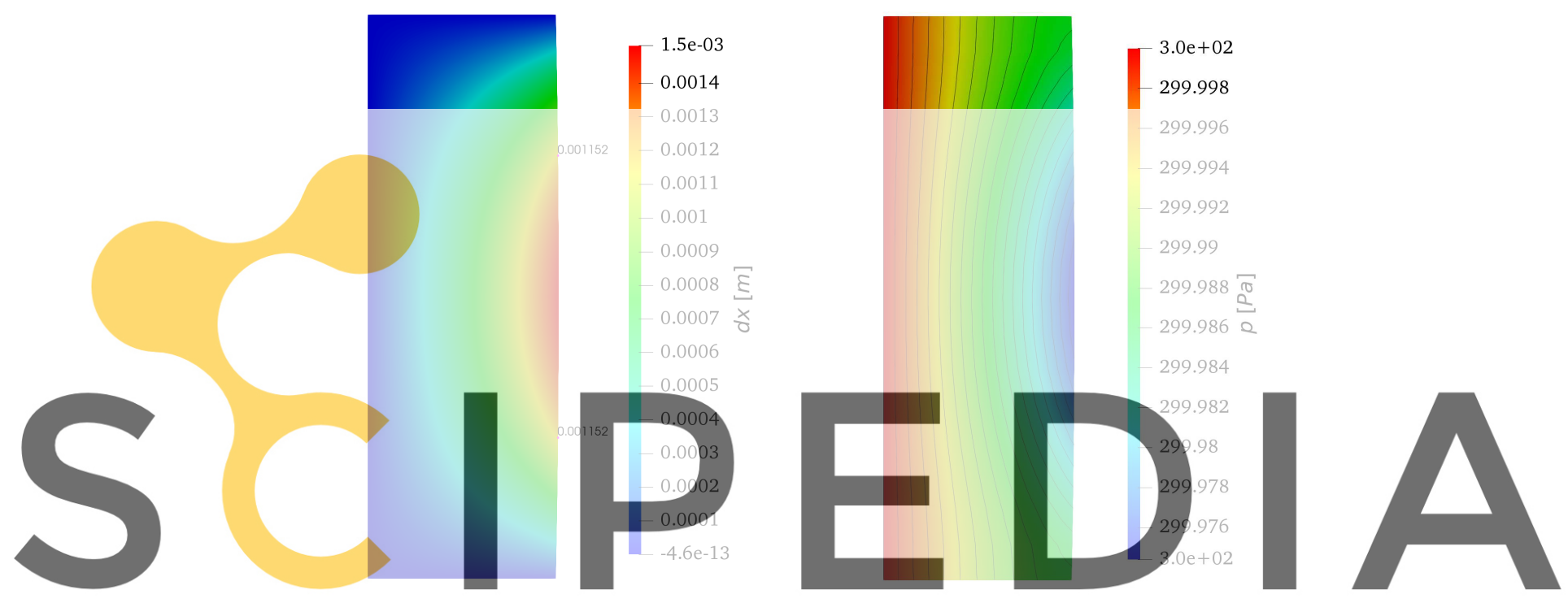

Figure 3: Airbag test: behavior of the displacement $\eta$ (center) and the pressure $p$ (right) in the reference case with

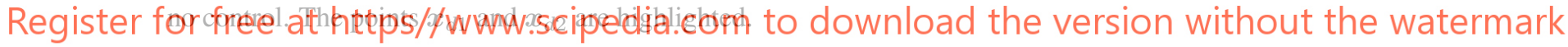

The goal is to control the displacement of the two points $\mathbf{x}_{\mathbf{d}_{\mathbf{1}}}$ and $\mathbf{x}_{\mathbf{d}_{2}}$, optimizing the pressure of the fluid on the boundary $\Gamma_{2}$. In order to break the symmetry of the problem, we choose a desired displacement of point $\mathbf{x}_{\mathbf{d}_{1}}$ equal to $\eta_{d_{1}}=-0.01 \mathrm{~m}$ and a desired displacement of $\mathbf{x}_{\mathbf{d}_{2}}$ equal to $\eta_{d_{2}}=0.01 \mathrm{~m}$. The functional associated with the presented numerical problem reads

$$
\mathcal{J}\left(\eta, p_{c}\right)=\frac{1}{2}\left(\left.\eta\right|_{\mathbf{x}_{\mathbf{d}_{\mathbf{1}}}}-\eta_{d_{1}}\right)^{2}+\frac{1}{2}\left(\left.\eta\right|_{\mathbf{x}_{\mathbf{d}_{\mathbf{1}}}}-\eta_{d_{2}}\right)^{2}+\frac{\lambda}{2} \int_{\Gamma_{2}} p_{c}^{2} d \Gamma .
$$

Many simulations have been done for different regularization parameter $\lambda$. In Figure 4, the control parameter $p$ obtained with different values of $\lambda$ is reported. Note that the trend obtained with $\lambda=10^{-10}$ shows slightly different values compared to the other two reported cases.

The reason of that is explained in Table 2, where the values of the functional $\mathcal{J}$ and of the displacements on $\mathbf{x}_{\mathbf{d}_{1}}$ and $\mathbf{x}_{\mathbf{d}_{2}}$ are reported for all the inspected $\lambda$ values. In fact, we can observe that the displacements obtained with $\lambda=10^{-8}$ and $\lambda=10^{-12}$ are similar and we are achieving the objective displacement 
Table 2: Objective functional $\mathcal{J}$, displacement $\eta$ and number of iteration obtained with different values of $\lambda$.

\begin{tabular}{ccccc}
\hline$\lambda$ & $\mathcal{J}\left(\eta, p_{c}\right)$ & $\eta_{\text {1opt }}[\mathrm{m}]$ & $\eta_{2 o p t}[\mathrm{~m}]$ & Iterations \\
\hline$\infty$ & $1.01327 \cdot 10^{-4}$ & 0.001152 & 0.001152 & - \\
$10^{-8}$ & $2.21289 \cdot 10^{-5}$ & -0.010695 & 0.005348 & 4 \\
$10^{-10}$ & $1.39504 \cdot 10^{-5}$ & -0.011603 & 0.013374 & 6 \\
$10^{-12}$ & $2.54909 \cdot 10^{-5}$ & -0.010032 & 0.004951 & 4 \\
\hline
\end{tabular}

$\eta_{d_{2}}$ from a lower value of it. On the contrary, if we consider the results obtained with $\lambda=10^{-10}$, we see that we are getting to the objective from a larger displacement. In fact, the functional has a different local minimum and the algorithm in this case moved to a different one. In Figure 4, we also reported the

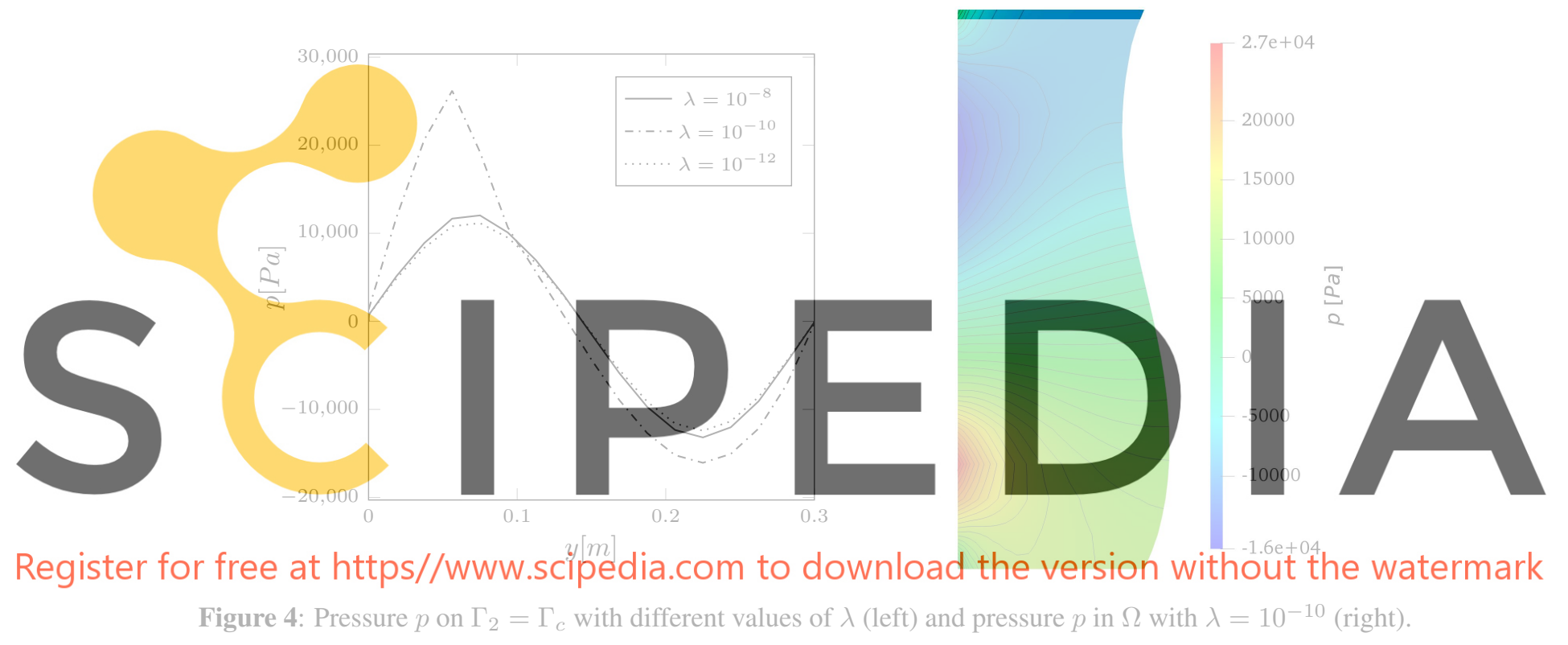

pressure of the fluid in $\Omega$ for $\lambda=10^{-10}$. Again, note that the value of $\lambda$ affects the pressure field to be imposed on the control domain $\Gamma_{2}$. In this case, the resulting velocity implies an inlet of fluid from a region of $\Gamma_{2}$ close to the point $\mathbf{x}_{\mathbf{d}_{2}}$ of which we want to increase the displacement, and an outlet of the fluid in a region of $\Gamma_{2}$ close to the point $\mathbf{x}_{\mathbf{d}_{1}}$, of which we want to reduce the displacement. In general, this case is an ill-posed control problem, due to various local minima close to each other.

\section{Conclusions}

In this work a mathematical and numerical method have been proposed to solve an optimal boundary control problem applied to a fluid-structure interaction model based on the Koiter's equation. Using this formulation, the equations for the solid become boundary conditions for the fluid equations reducing the computational cost of the numerical simulation. Then, we have obtained the optimality system applied to the pressure boundary control problem applied to the introduced Koiter FSI model to find the required 
memebrane displacement. We have introduced the steepest descent algorithm and solved the proposed control problem with a finite element code. Some simple numerical results have been presented to show the robustness of the multi-scale mathematical model.

\section{REFERENCES}

[1] Turek S and Hron J 2006 Lecture notes in computational science and engineering 53371

[2] Formaggia L, Quarteroni A and Veneziani A 2010 Cardiovascular Mathematics: Modeling and simulation of the circulatory system vol 1 (Springer Science \& Business Media)

[3] Bazilevs Y, Takizawa K and Tezduyar T E 2013 Computational fluid-structure interaction: methods and applications (John Wiley \& Sons)

[4] Le Tallec P and Mouro J 2001 Computer methods in applied mechanics and engineering 1903039 3067

[5] Cerroni D and Manservisi S 2016 Journal of Computational Physics 313 13-30

[6] Koiter W 1970 Proc. Int. Congr. of Mathematics, Nice vol 3 pp 123-130

[7] Nobile F and Vergara C 2008 SIAM Journal on Scientific Computing 30 731-763

[8] Gunzburger M D 2003 Perspectives in flow control and optimization vol 5 (Siam)

[9] Gunzburger M D and Manservisi S 2000 SIAM Journal on Numerical Analysis 37 1481-1512

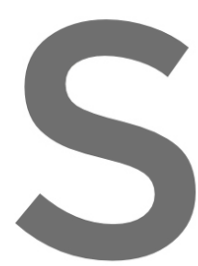

[10] Chireo L and Mansert

[11] Richter T and Wick

[12] Perego M, Veneziani A
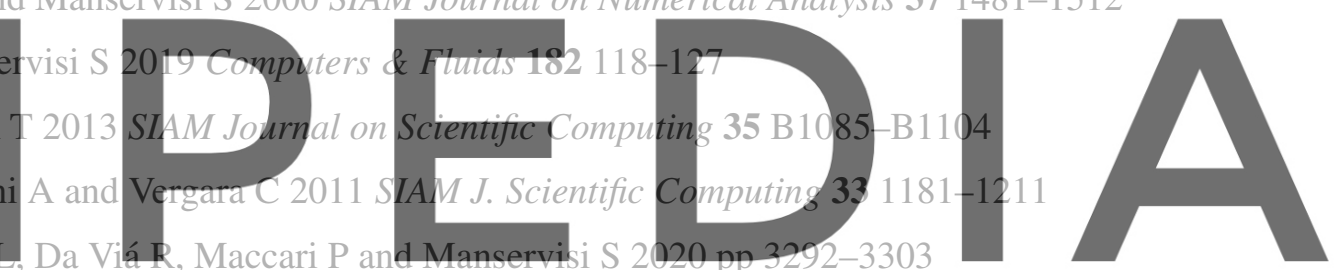

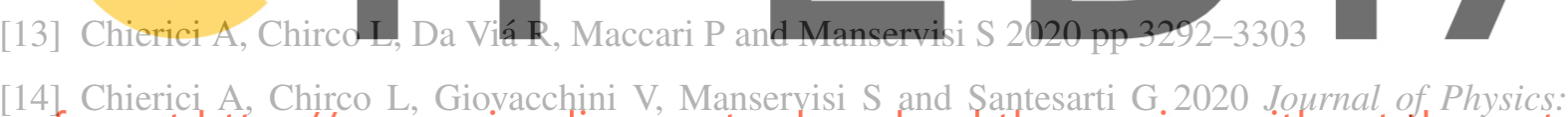

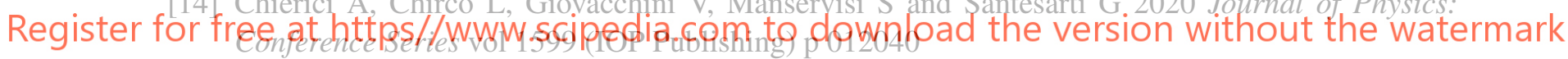

[15] Hughes T J, Liu W K and Zimmermann T K 1981 Computer methods in applied mechanics and engineering 29 329-349 